\section{Dominance of an UBA1 mutant clone over a CALR mutant clone: from essential thrombocytemia to VEXAS}

VEXAS (vacuoles, E1 enzyme, X-linked, autoinflammatory, somatic) is a recently described syndrome caused by somatic mutations in the UBA1 gene that lead to a multiorgan inflammatory disease and anemia. ${ }^{1}$ The clinical spectrum of UBA1-related diseases seems to be broader than the initial description, ${ }^{2,3}$ and the potential co-mutations occurring in UBA1-mutated patients have not been described yet. Here, we report the case of a patient with an initial CALR-mutated essential thrombocythemia (ET) who developed myelodysplasia cutis associated with an UBA1 mutation, with a concomitant extinction of both the CALR clone and the ET phenotype.

A 65-year-old man without major previous disease was diagnosed with ET and was treated with hydroxyurea and low dose aspirin. A CALR mutation was retrospectively identified in the diagnostic blood sample. After 10 years of treatment, at the age of 75 , he was referred for chronic edematous erythematous plaques on the chest, upper and lower limbs, suggestive of Sweet's syndrome, and a mild biological inflammatory syndrome (C-reactive protein at $30-50 \mathrm{mg} / \mathrm{L}$ ) (Figure $1 \mathrm{~A}$ ). Skin biopsies evidenced infiltration of myeloid cells with a histiocytoid morphology associated with incompletely segmented nuclei suggesting immature polymorphonuclear cells expressing CD163 and myeloperoxidase, which was consistent with the diagnosis of myelodysplasia cutis ${ }^{4}$ (Figure 1A). A targeted next generation sequencing (NGS) analysis covering 41 myeloid disease associated genes (ASXL1, ASXL2, ATM, BCOR, CALR, CBL, CEBPA, CSF3R, DDX41, DNMT3A, EZH2, FLT3, GATA2, HRAS, IDH1, IDH2, IKZF1, JAK2, KIT, KMT2A, KRAS, MPL, NF1, NPM1, NRAS, PHF6, PTPN11, RAD21, RUNX1, SETBP1,
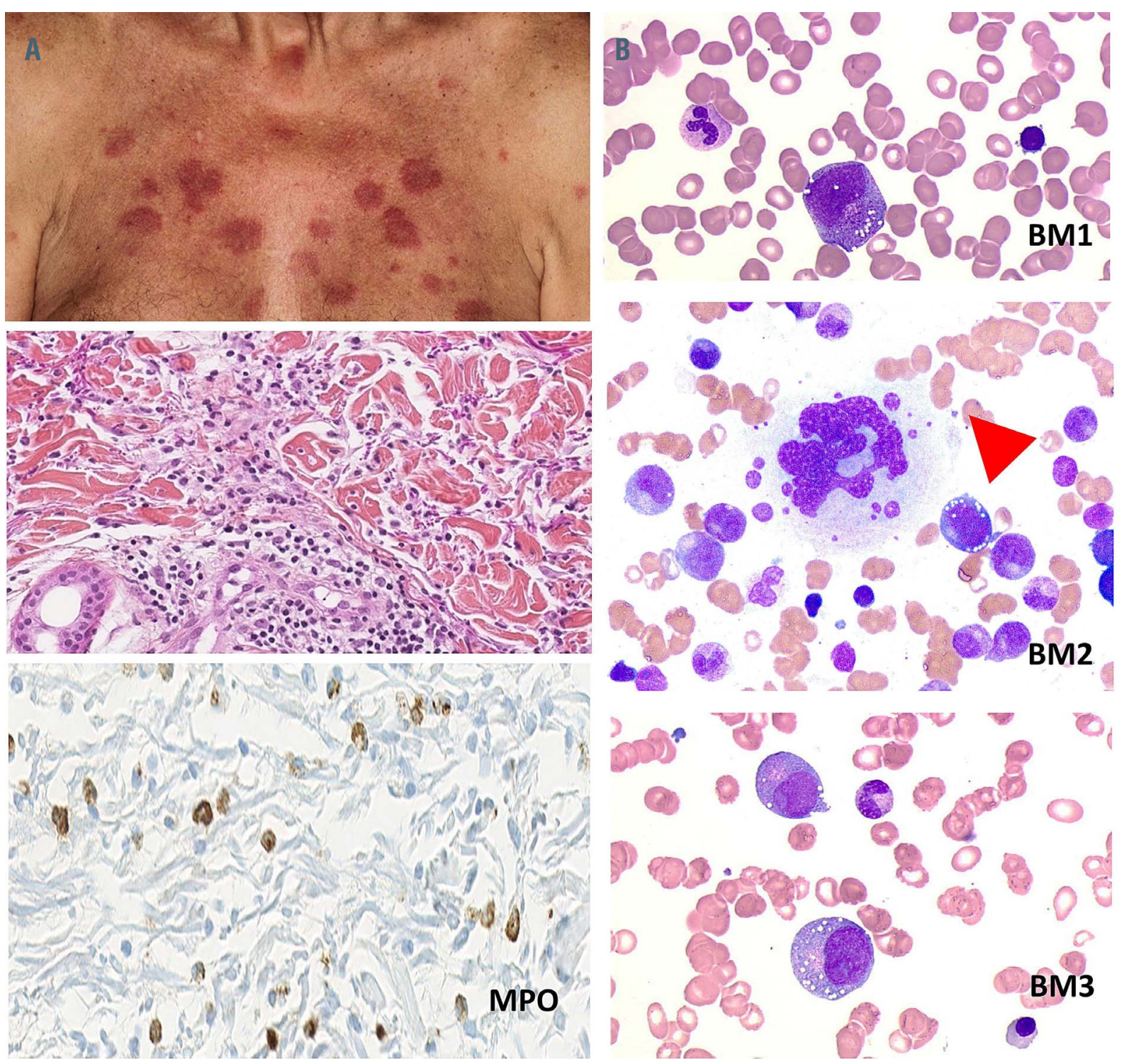

Figure 1. Skin and sequential bone marrow morphology. (A) Skin lesions and histopathological findings of skin biopsy, showing infiltration of histiocytoid cells staining positively for myeloperoxidase (MPO). (B) May-Grünwald-Giemsa stained bone marrow (BM) aspirate, exhibiting vacuolated myeloid precursors suggestive for a VEXAS syndrome (upper, BM1 after 10 years; middle, BM2 after 11 years; and lower, BM3 after 11.5 years of evolution after essential thrombocythemia diagnosis). 
A

\begin{tabular}{|c|c|c|c|c|}
\cline { 2 - 5 } \multicolumn{1}{c|}{} & \multirow{2}{*}{ SKIN } & \multicolumn{3}{|c|}{ BONE MARROW } \\
\cline { 3 - 5 } & $\begin{array}{c}\text { BM1 } \\
(10 \mathrm{y})\end{array}$ & $\begin{array}{c}\text { BM2 } \\
(11 \mathrm{y})\end{array}$ & $\begin{array}{c}\text { BM3 } \\
(11.5 \mathrm{y})\end{array}$ \\
\hline $\begin{array}{c}\text { DNMT3A } \\
\text { p.R882H }\end{array}$ & 22 & 38 & 47 & 41 \\
\hline $\begin{array}{c}\text { CALR } \\
\text { type I }\end{array}$ & 6 & 29 & 3 & 1 \\
\hline $\begin{array}{c}-7 \\
\text { (\% cells) }\end{array}$ & 30 & 2 & ND & ND \\
\hline
\end{tabular}

\section{B}
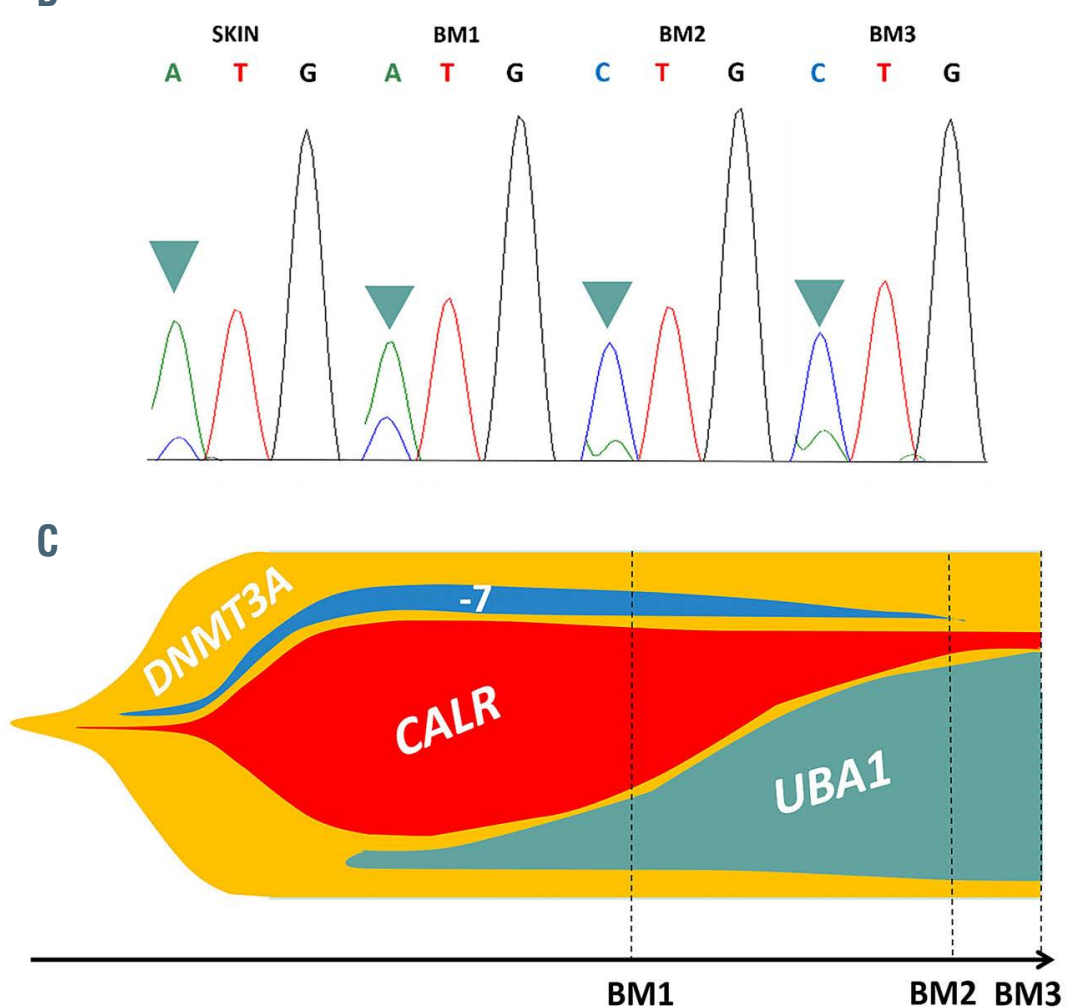

Figure 2. Clonal history of the two diseases. (A) Table of frequencies in bone marrow (BM) and skin biopsy: according to fluorescence in situ hybridization and next generation sequencing data. ND: not detected; $y$ : years. (B) Sanger sequencing electrophoregram, showing mosaic UBA1 c.121A $>C$ variant [NM_153280.3] in skin and successive three BM evaluations (BM1, 2, and 3). (C) Fish plot, representing clonal evolution upon BM evaluations (TET2 mutation is not displayed because of insufficient data in clonal evolution).

SF3B1, SH2B3, SMC1A, SMC3, SRSF2, STAG2, TET2, TP53, U2AF1, WT1, ZRSR2) was performed on the skin biopsy. Sequence analysis identified a DNMT3A p.R882H mutation (variant allele frequency [VAF] 22\%) and a CALR type 1 mutation (VAF 6\%). A monosomy of chromosome 7 was also identified by fluorescence in situ hybridization (FISH) in $30 \%$ of the cells of the biopsy (Figure 2A).

A concomitant bone marrow (BM) examination (BM1 after 10 years of evolution) showed evidence of megakaryocyte hyperplasia but neither signs of myelodysplasia nor blast excess. Using the same NGS panel, we found the same mutations of CALR (VAF $29 \%$ ) and DNMT3A (VAF $38 \%$ ), while FISH analysis detected only $2 \%$ of cells with monosomy 7 , suggestive of a clonal relation between granulocytes in the BM and in the skin lesions (Figure 2A). A thalidomide-based treatment was introduced, with an initial clinical improvement on skin lesions and a persistent normalization of platelet count, in spite of hydroxyurea progressive tapering and arrest. During this treatment, the patient progressively developed severe anemia, without efficacy of erythropoietin support, and needed repeated blood transfusion. Serial BM evaluations (BM2 after 11 years, and BM3 after 11.5 years of evolution) were performed, which still showed no evidence of myelodysplastic changes, but a decreased number of typical ET-related megakaryocytes and a progressive profound erythroblastopenia. Interestingly, DNMT3A mutation persisted at similar frequency, whereas $C A L R$ allele burden dropped to $1 \%$, and monosomy 7 became undetectable (Figure 2A).

After discontinuation of all therapies except aspirin, 
macrocytic anemia persisted, in association with a biological inflammatory syndrome with elevated C-reactive protein, and cutaneous lesions that led to a VEXAS hypothesis. Sanger sequencing identified an UBA1 variant with a high allele burden (p.Met41Leu, c.121A $>C$ [NM_153280.3]) (Figure 2B). Cytoplasmic vacuoles in erythroid and granulocytic precursors were also observed (Figure 1B). A retrospectively UBA1 mutation screening of the initial BM sample and skin biopsy detected the same variant at a lower allele burden, revealing the clonal sweep from the CALR mutated "ET clone" to the UBA1 "VEXAS" clone (Figure 2C). A treatment by ruxolitinib, the efficacy of which has been reported in VEXAS putatively due to its anti-inflammatory properties rather than a direct effect on the UBA1 mutated clone, was started and is currently ongoing with good improvement of cutaneous lesions. ${ }^{2}$ Hematopoietic stem cell transplantation was not considered due to the age of over 75 years at the time of VEXAS diagnosis.

A recent publication reports the newly described VEXAS syndrome, linked to somatic mutations in UBA1. Our case illustrates similar clinical manifestations with regard to the described entity, but the patient did not exhibit all the key clinical features like fever, pulmonary involvement, or polychondritis. This case suggests that UBA1 mutations can be associated to a diversity of clinical presentations and that the clinical spectrum of UBA1 related disease has to be refined. Other teams already described the frequent incomplete clinical presentation, and other not initially described involvement (vasculitis-like aspect or other) has also been reported. ${ }^{2,3,5,6}$

Sweet's syndrome was present in eight of $25(32 \%)$ participants from the first series of VEXAS syndrome. ${ }^{1}$ There are two forms of Sweet's syndrome: neutrophilic and histiocytoid. ${ }^{7,8}$ The histiocytoid subset, characterized by a dermal infiltration of immature myeloid cells with histiocytoid morphology, is associated with myelodysplastic syndromes in $24-31 \%$ of the cases. Some authors think that myelodysplasia cutis can be confirmed when the same cytogenetic abnormalities are found in the skin and BM of patients with histiocytoid Sweet's syndrome without leukemia, as in our patient. ${ }^{4}$ To our knowledge, this is the first report of myelodysplasia cutis associated with UBA1 mutations.

In our patient, we describe a progressive clonal replacement of a preexisting CALR-mutated ET clone, and illustrate the parallel clonal and phenotypic dynamics of two distinct driver-associated diseases. These results, thus demonstrate a clonal implication of different clones with a shared $D N M T 3 A$-mutated founder clone (Figure 2C). We assumed the association of DNMT3A and CALR mutations in the BM to be responsible for the initial ET. Then the association of the DNMT3A mutation, UBA1 mutation, and monosomy 7 , occurring in a subclone distinct from the DNMT3A and CALR mutant subclone, was associated with a skin tropism, with a minority of cells harboring monosomy 7 in the BM. Finally, the CALR clone was outcompeted by the UBA1 clone, which resulted in the clinical remission of the ET, and in the development of the VEXAS phenotype associating anemia, vacuolated myeloid precursors and systemic inflammation.

$C A L R$ mutations are strong driver mutations in myeloproliferative neoplasms. A decrease in allele burden can be observed during therapy such as interferon- $\alpha,{ }^{8}$ or in around half of the cases of secondary acute myeloid leukemia related to myeloproliferative neoplasms. ${ }^{9}$ In the present observation, clonal dominance conferred by the UBA1 mutation seems to be a major event during clonal evolution. This suggests that UBA1 mutations may provide a powerful selective advantage, able to overcome a $C A L R$ mutated clone. This might be due to a putative effect of UBA1 on the mutated hematopoietic stem cell proliferation and self-renewal, or might be in link with major changes induced by the autoinflammatory microenvironment that probably plays a role in shaping the clonal architecture, and might result in a selective advantage of the UBA1 clone over others.

In this observation, the role of hydroxyurea or thalidomide in clonal evolution cannot be excluded. It remains however unlikely, as the effects of hydroxyurea on CALR clones are often very limited, and the effects of thalidomide are known to be limited in thromboembolism cases. Moreover, expansion of preexisting clones after lenalinomide therapy, which is closely related to thalidomide or incidence of second malignancy after lenalinomide have not been clearly demonstrated. ${ }^{10-12}$

In conclusion, we describe the clonal dynamics of a $C A L R$ mutated subclone associated with ET, crushed by an UBA1 mutated subclone associated with myelodysplasia cutis and VEXAS, developing on a preexisting DNMT3A clonal hematopoiesis. This suggests somatic mutations of UBA1 can be associated with other mutations, and can be a secondary major driver event in clonal evolution. Common clinical features between VEXAS and hematological neoplasms with inflammatory manifestations could lead to a misdiagnosis. Extensive screening for UBA1 mutation is required to determine the real prevalence of VEXAS among patients with atypical presentation of myeloid neoplasms.

Mehdi Hage-Sleiman, ${ }^{1}$ Sophie Lalevée, ${ }^{2}$ Hélène Guermouche, ${ }^{1}$ Fabrizia Favale, ${ }^{1}$ Michael Chaquin, Maxime Battistella, ${ }^{3,4}$ Jean-David Bouaziz, ${ }^{1,4}$ Martine Bagot, ${ }^{2,4}$ François Delhommeau, ${ }^{1}$ Florence Cordoliani ${ }^{2}$ and Pierre Hirsch ${ }^{1}$

'Sorbonne Université, INSERM, Centre de Recherche SaintAntoine, AP-HP, Hôpital Saint-Antoine, Service d'Hématologie Biologique; ${ }^{2}$ Dermatology Department, Saint-Louis Hospital, AP-HP; ${ }^{3}$ Pathology Department, Saint-Louis Hospital, AP-HP and ${ }^{4}$ Université de Paris, Paris, France

Correspondence:PIERRE HIRSCH - pierre.hirsch@aphp.fr

doi:10.3324/haematol.2021.279418

Received: June 11, 2021

Accepted: July 21, 2021

Pre-published: July 29, 2021.

Disclosures: no conflicts of interest to disclose.

Contributions: MHS and PH performed molecular analyses and wrote the manuscript; SL, FC MB and JDB provided dermatologic data; MB performed histological analyses; FF performed molecular analyses; $M C$ and FD performed cytological analyses; HG performed cytogenetic and FISH analyses. All authors contributed to manuscript writing.

Funding: this work was supported by SiRIC CURAMUS (INCA-DGOS-Inserm_12560).

\section{References}

1. Beck DB, Ferrada MA, Sikora KA, et al. Somatic mutations in UBA1 and severe adult-onset autoinflammatory disease. N Engl J Med. 2020;383(27):2628-2638

2. Bourbon E, Heiblig M, Gerfaud-Valentin M, et al. Therapeutic options in Vexas syndrome: insights from a retrospective series. Blood. 2021;137(26):3682-3684

3. Oganesyan A, Jachiet V, Chasset F, et al. VEXAS syndrome: still 
expanding the clinical phenotype. Rheumatol Oxf Engl. 2021;60(9): e321-e323.

4. Osio A, Battistella M, Feugeas J-P, et al. Myelodysplasia cutis versus leukemia cutis. J Invest Dermatol. 2015;135(9):2321-2324.

5. Lee SMS, Fan BE, Lim JH-L, Goh LL, Lee JSS, Koh LW. A case of VEXAS syndrome manifesting as Kikuchi-Fujimoto disease, relapsing polychondritis, venous thromboembolism and macrocytic anaemia. Rheumatol Oxf Engl. 2021;60(9):e304-e306.

6. Poulter JA, Collins JC, Cargo C, et al. Novel somatic mutations in UBA1 as a cause of VEXAS syndrome. Blood. 2021:137(26):36763681.

7. Ghoufi L, Ortonne N, Ingen-Housz-Oro S, et al. Histiocytoid Sweet syndrome is more frequently associated with myelodysplastic syndromes than the classical neutrophilic variant: a comparative series of 62 patients. Medicine (Baltimore). 2016:95(15):e3033.

8. Verger E, Cassinat B, Chauveau A, et al. Clinical and molecular response to interferon- $\alpha$ therapy in essential thrombocythemia patients with CALR mutations. Blood. 2015;126(24):2585-2591.

9. Rampal R, Ahn J, Abdel-Wahab O, et al. Genomic and functional analysis of leukemic transformation of myeloproliferative neoplasms. Proc Natl Acad Sci U S A. 2014;111(50):E5401-E5410.

10. Palumbo A, Bringhen S, Kumar SK, et al. Second primary malignancies with lenalidomide therapy for newly diagnosed myeloma: a meta-analysis of individual patient data. Lancet Oncol. 2014; 15(3):333-342.

11. Jones JR, Cairns DA, Gregory WM, et al. Second malignancies in the context of lenalidomide treatment: an analysis of 2732 myeloma patients enrolled to the Myeloma XI trial. Blood Cancer J. 2016; 6(12):e506.

12. Kiladjian J-J, Rain J-D, Bernard J-F, Briere J, Chomienne C, Fenaux P. Long-term incidence of hematological evolution in three French prospective studies of hydroxyurea and pipobroman in polycythemia vera and essential thrombocythemia. Semin Thromb Hemost. 2006;32(4):417-421. 